\begin{tabular}{|c|l|}
\hline Title & $\begin{array}{l}\text { Reversed actrocytic GLT-1 during ischemia is crucial to excitotoxic death of neurons, but contributes to the survival of } \\
\text { astrocytes themselves. }\end{array}$ \\
\hline Author(s) & Kosugi, Tatsuro; Kawahara, Koichi \\
\hline Citation & $\begin{array}{l}\text { Neurochemical Research, 31/7), 933-943 } \\
\text { https://doi.org/10.1007/311064-006-9099-6 }\end{array}$ \\
\hline Issue Date & 2006-07 \\
\hline Doc URL & http://hdl.handle.net/2115/14610 \\
\hline Rights & The original publication is available at www.springerlink.com \\
\hline Type & article (author version) \\
\hline File Information & NR2006-31-7.pdf \\
\hline
\end{tabular}

Instructions for use 


\section{Reversed actrocytic GLT-1 during ischemia is crucial to excitotoxic death of neurons, but contributes to the survival of astrocytes themselves}

Tatsuro Kosugi and Koichi Kawahara

Laboratory of Cellular Cybernetics, Graduate School of Information Science and Technology, Hokkaido University, Sapporo 060-0814, Japan

Running title: Functional significance of reversed GLT-1 during ischemia

Address corresponding to:

Koichi Kawahara, $\mathrm{PhD}$

Professor of the Department of Cellular Cybernetics

Graduate School of Information Science and Technology

Hokkaido University

Sapporo 060-0814

Japan

TEL:+81-11-706-7591

FAX:+81-11-706-7591

E-mail: kawahara@cellc.ist.hokudai.ac.jp 


\begin{abstract}
During ischemia, the operation of astrocytic/neuronal glutamate transporters is reversed and glutamate and $\mathrm{Na}^{+}$are co-transported to the extracellular space. This study aims to investigate whether this reversed operation of glutamate transporters has any functional meanings for astrocytes themselves. Oxygen/glucose deprivation (OGD) of neuron/astrocyte co-cultures resulted in the massive death of neurons, and the cell death was significantly reduced by treatment with either AP5 or DHK. In cultured astrocytes with little GLT-1 expression, OGD produced $\mathrm{Na}^{+}$overload, resulting in the reversal of astrocytic $\mathrm{Na}^{+} / \mathrm{Ca}^{2+}$-exchanger (NCX). The reversed NCX then caused $\mathrm{Ca}^{2+}$ overload leading to the damage of astrocytes. In contrast, the OGD-induced $\mathrm{Na}^{+}$overload and astrocytic damage were significantly attenuated in PACAP-treated astrocytes with increased GLT-1 expression, and the attenuation was antagonized by treatment with DHK. These results suggested that the OGD-induced reversal of GLT-1 contributed to the survival of astrocytes themselves by releasing $\mathrm{Na}^{+}$with glutamate via reversed GLT-1.
\end{abstract}

Key words: ischemia, reversed GLT-1, astrocyte damage, $\mathrm{Na}^{+} / \mathrm{Ca}^{2+}$ exchanger, excitotoxic neuronal death 
List of Abbreviations

AP5: DL-2-Amino-5-phosphonopentanoic acid, a specific inhibitor of NMDA type glutamate receptors

CM: $\quad$ conditioned medium from cells cultured for more than 2 weeks

DHK: dihydrokainate, a specific blocker of GLT-1

EBSS: $\quad$ Earl's balanced salt solution

GFAP: $\quad$ glial fibrillary acidic protein

LDH: $\quad$ lactate dehydrogenase

MAP2: $\quad$ microtubule-associated protein 2

NCX: $\quad \mathrm{Na}^{+} / \mathrm{Ca}^{2+}$-exchanger

NADH: $\quad$ nicotinamide adenine dinucleotide

OGD: $\quad$ oxygen/glucose deprivation

PACAP: $\quad$ pituitary adenylate cyclase-activating polypeptide 


\section{INTRODUCTION}

Glutamate is a major excitatory neurotransmitter in the mammalian central nervous system. However, a high concentration of glutamate in the extracellular space leads to excitotoxic neuronal damage, and results in neuronal death (1). Therefore, in order to protect neurons from glutamate excitotoxicity, neurons and/or astrocytes must remove glutamate from the extracellular space mainly by using $\mathrm{Na}^{+}$-dependent glutamate transporters utilizing the $\mathrm{Na}^{+}$gradient across their plasma membranes (2). However, during ischemia, the activity of $\mathrm{Na}^{+}, \mathrm{K}^{+}$-ATPase is markedly suppressed and the ionic gradient of $\mathrm{Na}^{+}$is decreased (3), resulting in a reversal of the operation of astrocytic and neuronal glutamate transporters. Under these conditions, glutamate and $\mathrm{Na}^{+}$are transported from the intracellular to extracellular space (4). Previous studies have revealed that the ischemia-induced rise in the concentration of glutamate caused by this reversed uptake occurs mainly via astrocytic GLT-1 and is one of the causes of ischemia-induced neuronal death.

The uptake of glutamate by sodium-dependent glutamate transporters such as astrocytic GLT-1 is driven by the co-transport of three sodium ions and one proton with each glutamate ion into the cell, while one potassium ion is transported out of the cell (5-7). During ischemia, the activity of $\mathrm{Na}^{+}, \mathrm{K}^{+}$-ATPase is markedly suppressed, resulting in the accumulation of intracellular sodium ions (8). Under such conditions, the operation of GLT-1 is reversed, and three sodium ions are thus co-transported with a glutamate ion out of the cell (9). It has been postulated that the excessive influx of $\mathrm{Na}^{+}$ into astroglial cells causes significant astroglial cell death (10) due to $\mathrm{Ca}^{2+}$ overload probably via a reversed $\mathrm{Na}^{+} / \mathrm{Ca}^{2+}$ exchanger. Therefore, there is a possibility that the 
ischemia-induced reverse mode operation of GLT-1 contributes to the survival of astrocytes themselves by reducing $\mathrm{Na}^{+}$overload, although the reversal causes massive neuronal death.

In this study, we investigated whether this reverse mode operation of astrocytic GLT1 during ischemia has any functional meaning for astrocytes themselves. Here we show that the ischemia-induced reverse mode operation of astrocytic GLT-1 was toxic to neurons but contributed to the survival of astrocytes themselves by maintaining their $\mathrm{Na}^{+}$gradient across cell membranes. This is probably one of the reasons why astrocytes are more resistant to severe ischemic insult than neurons. 


\section{EXPERIMENTAL PROCEDURE}

\section{Neuron/astrocyte co-culture}

Culture methods were described previously in detail (11-13). In brief, neurons/astrocytes were prepared from 17-day-old embryonic rat cortices and grown in Dulbecco’s modified Eagle’s medium (DMEM, Gibco, Grand Island, NY) which was supplemented with 10\% heat-inactivated fetal bovine serum (FBS), 10\% Ham's F12, and $0.24 \%$ penicillin/streptomycin (culture medium) and maintained in a $5 \% \mathrm{CO}_{2}$ incubator at $37^{\circ} \mathrm{C}$. The cultures were fed a conditioned medium (CM) twice a week. To obtain the CM, cells from the 17-day-old embryonic rat cortices were plated onto poly-L-lysine-coated 6-well dishes and cultured for more than 2 weeks. The cultures were then fed a cooled culture medium and incubated for an additional day. After 13-15 days, the neurons in these cultures sit on the top of a confluent monolayer of astrocytes. The experiments were performed using these cultures.

\section{Astrocyte-enriched culture}

Culture methods were described previously in detail (11). In brief, cultures were prepared from cortical astrocytes of postnatal 2-day-old rat pups. Cortical hemispheres were removed, cleaned, and dissociated using papain and mechanical trituration. Cells were placed on poly-L-lysine-coated glass coverslips and maintained in the culture medium. The medium was changed $48 \mathrm{~h}$ later and twice a week thereafter. The experiments described here were performed on astrocytes maintained for 10-13 days in culture. 


\section{Immunocytochemistry}

Astrocytes and neurons were identified by immunostaining with antibodies against glial fibrillary acidic protein (GFAP; Sigma, St Louis, MO) and microtubule-associated protein 2 (MAP-2; Sigma), respectively. The astrocytic glutamate transporter GLT-1 was detected by immunostaining with anti-GLT-1 polyclonal antibodies (Chemicon, Temecula, CA). For the labeling of MAP-2, GFAP, and GLT-1, the cortical cells were fixed with $4 \%$ paraformaldehyde for 5 minutes at $4{ }^{\circ} \mathrm{C}$, followed by $95 \%$ methanol in PBS for 10 minutes at $-20^{\circ} \mathrm{C}$. The cells were then incubated with a primary antibody over a $24 \mathrm{~h}$ period using a dilution of 1:1000 for MAP-2, 1:400 for GFAP, and 1:2500 for GLT-1. After washing with phosphate-buffered saline (PBS), the cells were incubated with a secondary antibody containing $1.0 \%$ goat serum for 30 minutes. For labeling, a 1:500 dilution of biotinylated goat antibody against mouse or guiniea pig IgG (Vector Laboratories, Burlingame, CA) was used. Bound antibodies were detected by the avidin-biotin-peroxidase complex (ABC) method, using a commercial ABC kit (Vector Laboratories). Observation of the peroxidase activity was made possible by incubation with $0.1 \%$ 3,3'-diaminobenzidine tetrahydrochloride (DAB) in a $50 \mathrm{mM}$ Tris-HCl buffer (pH 7.4) supplemented with $0.02 \% \mathrm{H}_{2} \mathrm{O}_{2}$.

\section{Oxygen-Glucose deprivation}

Cortical cultures were subjected to oxygen-glucose deprivation (OGD) injury using a protocol described previously (12-14). In brief, cultures were placed in an anaerobic 
chamber containing the deoxygenation reagent and glucose-free Earl’s balanced salt solution (EBSS: $116 \mathrm{mM} \mathrm{NaCl}, 0.8 \mathrm{mM} \mathrm{MgSO}_{4}, 5.4 \mathrm{mM} \mathrm{KCl,} 1.0 \mathrm{mM} \mathrm{NaH} \mathrm{PO}_{4}, 26.2$ $\mathrm{mM} \mathrm{NaHCO}_{3}, 1.8 \mathrm{mM} \mathrm{CaCl}_{2}, 0.01 \mathrm{mM}$ glycine, and $10 \mathrm{mg} / \mathrm{l}$ ) was added. Near anoxic conditions were achieved using an Anaero-Pack System (Mitsubishi Gas Chemical, Tokyo, Japan). To terminate OGD, cultures were carefully washed with glucose (20 $\mathrm{mM}$ ) containing EBSS, and then incubated again in culture medium at $37{ }^{\circ} \mathrm{C}$ in $95 \%$ air-5\% $\mathrm{CO}_{2}$ (reperfusion). Cultures with sham treatment not deprived of oxygen and glucose were placed in EBSS containing $20 \mathrm{mM}$ glucose.

\section{Western Blot analysis.}

Cell lysates were diluted 3:1 in sample buffer (187.5 mM Tris-HCl containing 6\% SDS, and 15\% glycerol, 15\% 2-mercaptoethanol) and denatured at $100{ }^{\circ} \mathrm{C}$ for $3 \mathrm{~min}$. Proteins were electrophoresed on a 10\% SDS-polyacrylamide gel and transferred onto a polyvinylidene difluoride membrane (Bio-Rad, Hercules, CA). Nonspecific binding sites were blocked with $5 \%$ nonfat milk for $60 \mathrm{~min}$, and then the membrane was incubated overnight at $4{ }^{\circ} \mathrm{C}$ with one of the following antibodies: polyclonal antibody to GLT-1 (1:10000) or monoclonal antibody to actin (1:2000) (Sigma), and then horseradish peroxidase-labeled anti-guinea pig for GLT-1 (1:2000) (Cell Signaling Technology) or anti-mouse IgG antibody for actin (1:2000) (Cell Signaling Technology), respectively. The immunoreactive bands were detected with an enhanced chemiluminescence kit (NEN Life Science Products, Boston, MA). Quantification of the levels was preformed by densitometric analysis using Scion Image Beta 4.02 (Scion Corporation). 


\section{Measurement of the extracellular glutamate concentration}

The extracellular concentration of glutamate (Glu) was measured using an enzymatic assay $(13,15-16)$. In the presence of Glu and $\beta$-nicotinamide adenine dinucleotide $\left(\mathrm{NAD}^{+}\right)$, L-glutamic dehydrogenase (GDH) produces $\alpha$-ketoglutarate and NADH, a product that fluoresces when excited at $360 \mathrm{~nm}$. In the presence of $\alpha$-ketoglutarate and L-alanine, glutamate pyruvate transaminase (GPT) produces L-glutamate. Therefore, GDH (50 U/ml), NAD ${ }^{+}$(2 mM), GPT (4 U/ml), and L-alanine (2 mM) were added to the external solution, and the fluorescence was excited at $360 \mathrm{~nm}$ and detected at $>510$ nm with a fluorescent microscope. Images excited at $360 \mathrm{~nm}$ were acquired with integration times of $5 \mathrm{~s}$ at intervals of $50 \mathrm{~s}$. The extracellular Glu was detected as an increase in NADH fluorescence. This method does not necessarily reflect the quantitative extracellular Glu level, but can detect subtle changes in the concentration of Glu. Fluorescent images were acquired with a cooled CCD camera (C4880-80; Hamamatsu Photonics, Hamamatsu, Japan). An analysis of the acquired images was conducted with an image processing and measuring system (AQUACOSMOS; Hamamatsu Photonics). The pseudo-color image representation of NADH fluorescence reflecting the extracellular concentration of glutamate was used. The fluorescence intensity increases from dark blue to red through yellow.

\section{Measurement of the intracellular $\mathrm{Na}^{+}$and $\mathrm{Ca}^{2+}$ concentration}

Intracellular $\mathrm{Na}^{+}$and $\mathrm{Ca}^{2+}$ were measured in astrocytes after loading the cells with the $\mathrm{Na}^{+}$-sensitive fluorescent dye sodium-binding benzofuran isophthalate (SBFI-AM) and Fluo-4, respectively. Cell loading was performed using $22 \mu \mathrm{M}$ SBFI/AM in 
conditioned medium supplemented with $0.03 \%$ Pluronic F-127 and after 6 hours, dishes were washed twice using EBSS, and $5 \mu \mathrm{M}$ Fluo- 4 was loaded for 1 hour. The fluorescence was sequentially excited at $340 \mathrm{~nm}$ (for SBFI), $380 \mathrm{~nm}$ (for SBFI) and 488 $\mathrm{nm}$ (for Fluo-4) and detected at > $510 \mathrm{~nm}$ by a fluorescent microscope (Olympus, 1X70). Pairs of $340 \mathrm{~nm}$ and $380 \mathrm{~nm}$ images were acquired with integration times of $478 \mathrm{~ms}$ at intervals of $150 \mathrm{~s}$ and analyzed off-line (AQUACOSMOS; Hamamatsu Photonics). The change in intracellular $\mathrm{Na}^{+}$and $\mathrm{Ca}^{2+}$ was estimated from the fluorescence excitation ratio $\left(\mathrm{F}_{340} / \mathrm{F}_{380}\right)$ and $\left(\mathrm{F} / \mathrm{F}_{0}\right)$, respectively.

\section{Evaluation of astrocytic damage}

The astrocytic damage induced by OGD was confirmed by measuring the amount of lactate dehydrogenase (LDH) released into the solution. LDH activity was measured by a method based on the reduction of nitroblue tetrazolium to diformazan by NADH. In the presence of $\mathrm{LDH}, \mathrm{NAD}^{+}$and lactate produce pyruvate and NADH. This NADH reduces nitroblue tetrazolium to diformazan. At $20 \mathrm{~h}$ after the OGD insult, $50 \mu \mathrm{l}$ of culture medium was transferred from each dish and the enzymic reaction was begun with the addition of $50 \mu \mathrm{l}$ of reaction mixture supplied with the LDH-Cytotoxic test WAKO (Wako, Japan). The reaction was run at room temperature for $30 \mathrm{~min}$ and was terminated by the addition of $100 \mu \mathrm{l}$ of $0.5 \mathrm{M}-\mathrm{HCl}$. The amount of diformazan produced was determined by measuring absorbance at a wavelength of $560 \mathrm{~nm}$ with an Ultrospec 3300 pro (Amersham Pharmacia biotech.). Two dishes were treated with $1 \mathrm{ml}$ of 1\% triton-X 100 in CM and the average level of LDH activity was taken as maximal LDH release. Then released LDH activity from all dish was divided by maximal LDH release to calculate percent cell death in each dish. 


\section{Survival rate of neurons}

Neuronal death was analyzed following observation of the nuclear morphology using the fluorescent DNA-binding dyes, Hoechst 33342 (H33342) and propidium iodide (PI). Individual nuclei were observed using a fluorescent microscope (Olympus, IX70, Tokyo, Japan) and subsequently analyzed. PI was used to identify nonviable cells. More specifically, an average of 450-500 neurons from random fields were analyzed in each experiment. The survival rate of neurons - meaning the percentage of viable neurons remaining - was determined by placing images of nuclear staining on phasecontrast images, and calculating (viable neurons/total neurons before drug treatment) $\times$ 100. At least 4 independent experiments $(n \geq 4)$ were conducted and analyzed.

\section{Statistics}

The data are expressed as the mean \pm S.D. Comparisons were performed using the oneway analysis of variance (ANOVA) followed by a paired $t$ test. A $P$ value of less than 0.01 was considered statistically significant. 


\section{RESULTS}

We first investigated whether the reversed operation of astrocytic GLT-1 contributed to the ischemia-induced, NMDA receptor-mediated excitotoxic death of neurons using mixed neuron/astrocyte cultures (from E17 Wistar rats and days in vitro 13-16; See Methods). Brain ischemia was simulated by depriving the cultures of both oxygen and glucose (OGD) (12-14). Immunostaining of the cultures using either an antimicrotuble-associated protein-2 (MAP-2) or an anti-glial fibrillary acidic protein (GFAP) antibody demonstrated that MAP-2-positive neurons (Fig. 1A1) and GFAPpositive astrocytes co-existed in the cultures (Fig. 1A2), indicating that the cultures were mixed. Immunostaining using an anti-GLT-1 antibody revealed that cultured astrocytes in the mixed neuron/astrocyte cultures expressed GLT-1 (Fig. 1A3). GLT-1positive astrocytes were preferentially identified in astrocytes just underneath the aggregate of neurons similar to our previous observations (11). OGD of mixed cultures for 90 min resulted in the massive death of neurons $24 \mathrm{~h}$ after the insult (Fig. 1C and F), and the cell death was significantly reduced by treatment with DL-2-Amino-5phosphonopentanoic acid (AP5 $100 \mu \mathrm{M}$ ), a specific blocker of NMDA receptors (Fig. 1D and F). The OGD-induced death of neurons was also significantly reduced by treatment with dihydrokainate (DHK, $200 \mu \mathrm{M}$ ) during OGD (Fig. 1E and F). Although OGD caused significant death of neurons, almost all of the astrocytes seemed to remain intact under these experimental conditions. Red nuclei in C3 indicate propidium iodide (PI)-positive dead neurons. Many PI-positive neurons were identified, but few PIpositive astrocytes were observed (Fig. 1C3). It is generally believed that astrocytes are less susceptible to ischemic injury and subsequent metabolic inhibition than neurons (17). Thus, there is a possibility that the reverse mode operation of astrocytic GLT-1 
during ischemia has some functional meanings for astrocytes themselves. We therefore investigated this issue using astrocyte-enriched cultures.

Previous studies have revealed that the expression level of GLT-1 is regulated by several factors like epidermal growth factor (EGF) (18) or transforming growth factor- $\alpha$ (TGF- $\alpha$ ) (19) in cultured astrocytes. In addition, pituitary adenylate cyclase-activating polypeptide (PACAP), a neuron-derived peptide, has been identified as crucial for upregulating GLT-1 expression when glial cultures are treated with neuron-conditioned medium (20). These findings seem to support our previous immunocytochemical finding that GLT-1-positive astrocytes were preferentially identified just underneath aggregates of neurons in neuron/astrocyte co-cultures (11). Thus, we first analyzed the changes in the expression of GLT-1 in astrocyte-enriched cultures treated with PACAP (1 $\mu \mathrm{M})$, a neuron-derived peptide, for $24 \mathrm{~h}$. An immunocytochemical analysis using an anti-MAP-2 antibody showed that MAP-2-positive neurons were not present (Fig. 2A2), and almost all the cells were stained by an anti-GFAP antibody in our astrocyteenriched cultures (Fig. 2A1). In the astrocyte-enriched cultures, few GLT-1-positive astrocytes were observed (Fig. 2A3). Treatment with PACAP for 24 h markedly increased the astrocytic GLT-1 expression (Fig. 2A4). Western blot analyses also revealed that PACAP treatment significantly increased the expression of astrocytic GLT-1 (Fig. 2E1-F2). We then investigated whether the extracellular concentration of glutamate increased during OGD caused by the reversal of GLT-1's function in the PACAP-treated cultures. In the sham-treated cultures with little GLT-1 expression, the extracellular glutamate did not increase significantly during OGD for 4 h (Fig. 2B, G, H). In contrast, the extracellular glutamate markedly increased during OGD in the PACAP-treated cultures with GLT-1 expression (Fig. 2C, G, H), and the OGD-induced 
increase in glutamate was significantly suppressed by treatment with DHK (400 $\mu \mathrm{M})$, a selective blocker of astrocytic GLT-1 (6, 21-22) (Fig. 2D, G, H).

We then investigated whether the reverse mode operation of astrocytic GLT-1 during ischemia contributed to the survival of astrocytes themselves. OGD for $4 \mathrm{~h}$ resulted in the damage of sham-treated astrocytes with little GLT-1 expression $20 \mathrm{~h}$ after the end of OGD, but the cell damage caused by the same insult was significantly suppressed in PACAP-treated astrocytes with GLT-1 expression (Fig. 3A). The reduction of cell damage in PACAP-treated astrocytes was reversed by treatment with DHK (400 $\mu \mathrm{M})$ during OGD. Treatment of sham-treated astrocytes without GLT-1 with DHK (400 $\mu \mathrm{M})$ during OGD did not significantly change the cell damage (Fig. 3B). Treatment of cultures with DHK without OGD did not induce significant cell damage. These results suggested that the OGD-induced reverse mode operation of astrocytic GLT-1 contributed to the survival of astrocytes themselves.

The question then arises as to what mechanisms are involved in the increased resistance to OGD observed in PACAP-treated astrocytes with increased GLT-1 expression. We next investigated the possibility that the ischemia-induced reverse mode operation of GLT-1 contributes to the survival of astrocytes themselves by reducing $\mathrm{Na}^{+}$overload, although the reversal paradoxically causes massive neuronal death. The fluorescence intensity in pseudo-color images in Fig.4 increases from dark blue to red through yellow. In the sham-treated cultures with little GLT-1 expression, the intracellular concentration of both $\mathrm{Na}^{+}$and $\mathrm{Ca}^{2+}$ markedly increased during $4 \mathrm{~h}$ OGD (Figs. 4A3, 4A5, and Fig. 5). In contrast, the OGD-induced rise in the intracellular concentration of both $\mathrm{Na}^{+}$and $\mathrm{Ca}^{2+}$ in the PACAP-treated cultures with 
GLT-1 expression was significantly reduced as compared with that in the sham-treated cultures (Figs. 4B3, 4B5, and Fig. 5), and this reduction was significantly reversed by treatment with DHK (400 $\mu \mathrm{M})$ (Fig. 4C3, 4C5, and Fig. 5). When the fluorescence indicators weren't loaded into astrocytes in the sham- or PACAP-treated astrocyteenriched cultures, each emission $\left(\mathrm{F}_{340} / \mathrm{F}_{380}, \mathrm{~F}_{488}\right)$ did not change significantly during $4 \mathrm{~h}$ OGD (Fig 5A1 and B1). These results suggested that OGD resulted in the marked rise in the concentration of intracellular $\mathrm{Na}^{+}\left(\mathrm{Na}^{+}\right.$overload) in cultured astrocytes without GLT-1 expression, but the rise in intracellular $\mathrm{Na}^{+}$was significantly reduced due to the OGD-induced reversal of GLT-1's function in PACAP-treated astrocytes with GLT-1 expression. In addition, OGD-induced $\mathrm{Na}^{+}$overload then resulted in a significant rise in the intracellular concentration of $\mathrm{Ca}^{2+}\left(\mathrm{Ca}^{2+}\right.$ overload) in cultured astrocytes, suggesting that the operation of NCX was reversed by the OGD-induced $\mathrm{Na}^{+}$overload. We next test this possibility. Treatment with KB-R7943 (5 $\mu \mathrm{M})$, an inhibitor of the reversed NCX (23), significantly suppressed the OGD-induced rise in the intracellular concentration of $\mathrm{Ca}^{2+}$ (Fig. 4D5), but did not suppress the rise in $\mathrm{Na}^{+}$in the sham-treated astrocytes (Fig. 2D3). In addition, the OGD-induced damage in sham-treated astrocytes was also reduced by treatment with KB-R7943 (5 $\mu \mathrm{M})$ (Fig. 5C), suggesting that the reversed operation of NCX was responsible for the damage. 


\section{DISCUSSION}

The maintenance of intracellular concentration of $\mathrm{Na}^{+}$at a low level in astrocytes is critical to a variety of astrocytic functions. Once the $\left[\mathrm{Na}^{+}\right]_{\mathrm{i}}$ gradient across plasma membrane is collapsed caused by the decreased activity of $\mathrm{Na}^{+} / \mathrm{K}^{+}$ATPase under ischemic condition, $\mathrm{Ca}^{2+}$ homeostasis attained mainly by using $\mathrm{Na}^{+} / \mathrm{Ca}^{2+}$ exchanger (NCX) is compromised. The modulation of astrocytic $\left[\mathrm{Ca}^{2+}\right]_{\mathrm{i}}$ has been implicated in the control of astrocyte-neuron signaling (24), proliferation and differentiation (25), $\mathrm{K}^{+}$ channel activities (26), and of the astrocytic release of neurotransmitter (27). The dysfunction of astrocytic $\mathrm{Ca}^{2+}$ homeostasis results in $\mathrm{Ca}^{2+}$ overload and astrocytic cell death. In astrocytes, there are at least two routes of calcium influx during ischemia. One is via the activation of voltage-sensitive $\mathrm{Ca}^{2+}$ channels and the other is via the reverse-mode operation of NCX. Both of them result from the accumulation of intracellular $\mathrm{Na}^{+} . \mathrm{Ca}^{2+}$ influx via reversed NCX seems to be the primary cause of ischemia-induced death of astrocytes. In fact, in this study, the inhibition of NCX during in vitro ischemia by treatment of cultures with KB-R7943, a potent specific inhibitor of the reversed NCX at the low concentration of $5 \mu \mathrm{M}$ (28), suppressed the rise in $\left[\mathrm{Ca}^{2+}\right]_{\mathrm{i}}$ and ischemia-induced cell damage (Figs. 4 and 5C). These results suggest that ischemia-induced death of astrocytes is mainly caused by $\mathrm{Ca}^{2+}$ overload via the reversed-mode operation of NCX, and that in normal condition astrocytes suppress the rise in $\left[\mathrm{Ca}^{2+}\right]_{\mathrm{i}}$ by using the steep $\mathrm{Na}^{+}$gradient across their cell membranes.

A previous study has revealed that neuronal cells are injured caused by glutamate efflux via reversed astrocytic glutamate transporter GLT-1 during brain ischemia (29). In the present study, treatment with either AP5 or DHK during OGD suppressed the 
OGD-induced neuronal death (Fig. 1B-F). These results suggested that neurons were degenerated by NMDA-type glutamate receptor-mediated glutamate toxicity, and that the primary source of glutamate during OGD was astrocytic GLT-1. Therefore, the present findings have led to an idea that the ischemia-induced reverse mode operation of GLT-1 contributes to the survival of astrocytes themselves by reducing $\mathrm{Na}^{+}$overload, although the reversal paradoxically causes massive neuronal death.

The intracellular $\mathrm{Na}^{+}$accumulation in astrocytes is also known to result in the reversed operation of astrocytic glutamate transporters. To date, two astrocytic glutamate transporters GLT-1 and GLAST have been identified in cortical astrocytes. It is generally believed that astrocytic GLT-1 plays a dominant role both in the uptake (30-31) and release (29) of glutamate in the brain. In our astrocyte-enriched culture, few GLT-1-positive astrocytes were identified (Fig. 2A3). A previous study has demonstrated that astrocytic GLT-1 is up-regulated by neuron-derived factors such as a pituitary adenylate cyclase-activating polypeptide (PACAP) (20). Thus, in the present study, we treated astrocyte-enriched cultures with PACAP $(1 \mu \mathrm{M}, 24 \mathrm{~h})$ to express GLT-1. PACAP-treated astrocytes with GLT-1 expression showed resistance to ischemic death, and maintained the intracellular concentration of both $\mathrm{Na}^{+}$and $\mathrm{Ca}^{2+}$ at low levels during in vitro ischemia (Figs. 3-5). This protective effect disappeared by the inhibition of GLT-1 with DHK (Fig. 3). Therefore, these findings suggested that the co-transport of $\mathrm{Na}^{+}$and glutamate via reversed GLT-1 during ischemia contributed to the suppression of the reversed NCX-induced rise in $\left[\mathrm{Ca}^{2+}\right]_{\mathrm{i}}$ by maintaining $\mathrm{Na}^{+}$ gradient across astrocytic plasma membranes, leading to the increased resistance against ischemic cell death.

In the present study, intracellular $\mathrm{Na}^{+}$and $\mathrm{Ca}^{2+}$ were measured in astrocytes with 
SBFI-AM and Fluo-4-AM, respectively. The excitation wavelength was 340 nm, 380 $\mathrm{nm}$ (for SBFI) and $488 \mathrm{~nm}$ (for Fluo-4) and emission was detected at $>510 \mathrm{~nm}$ (for every excitation). These filter settings were the same as those used for monitoring NADH fluorescence. Thus, there was a possibility that the fluorescence signal of each indicator might not exactly reflect the intracellular changes in $\mathrm{Na}^{+}$and $\mathrm{Ca}^{2+}$, since the signal possibly contained the changes in intracellular NADH fluorescence. However, when the fluorescence indicators weren’t loaded into astrocytes in the sham- or PACAP-treated astrocyte-enriched culture, each emission $\left(\mathrm{F}_{340} / \mathrm{F}_{380}, \mathrm{~F}_{488}\right)$ did not change significantly during 4 h OGD (Fig 5A1 and B1). Therefore, the potential changes in NADH fluorescence did not affect the fluorescence signal reflecting either intracellular $\mathrm{Na}^{+}$or $\mathrm{Ca}^{2+}$.

It is generally believed that astrocytes are less susceptible to ischemic injury and subsequent metabolic inhibition than neurons (17), in part because they possesses larger glycogen stores, lower density expression of voltage-sensitive $\mathrm{Na}^{+}$channels (32), and less susceptibility to glutamatergic excitotoxicity (33). Takahashi et al. (10) postulated that such a difference in the susceptibility to ischemia between neurons and astrocytes might be related to less $\mathrm{Na}^{+}$influx into astrocytes as compared with neurons during ischemia, since astrocytes do not generate an action potential upon membrane depolarization. The present study provides a novel finding capable of explaining one of the reasons why astrocytes are more resistant to severe ischemic insult than neurons. 


\section{ACKNOWLEDGEMENTS}

The authors wish to thank Dr. Takayuki Nakajima, Research Institute for Electronic Science, Hokkaido University, for advice on the immunostaining of cortical cultures. 


\section{REFERENCES}

1. Choi, D.W. 1998. Glutamate neurotoxicity and diseases of the nervous system. Neuron 1:623-634.

2. Rothstein, J.D., Martin, L., Levey, A.I., Dykes-Hoberg, M., Jin, L., Wu, D., Nash, N. and Kuncl, R.W. 1994. Localization of neuronal and glial glutamate transporters. Neuron 13:713-725.

3. Lees, G.J. 1991. Inhibition of sodium-potassium-ATPase: a potentially ubiquitous mechanism contributing to central nervous system neuropathology. Brain Res. Rev. $16: 283-300$

4. Szatkowski, M., Barbour, B., and Attwell, D. 1990. Non-vesicular release of glutamate from glial cells by reversed electrogenic glutamate uptake. Nature 348:443-446.

5. Phillis, J.W., Smith-Barbour, M., Perkins, L.M. and O’Regan, M.H. 1994. Characterization of glutamate, aspartate, and GABA release from ischemic rat cerebral cortex. Brain Res. Bull. 34:457-466.

6. Levy, L.M., Warr, O., and Attwell, D. 1998. Stoichiometry of the glial glutamate transporter GLT-1 expressed inducibly in a Chinese hamster ovary cell line selected for low endogenous $\mathrm{Na}^{+}$-dependent glutamate uptake. J. Neurosci. 18:9620-9628. 
7. Anderson, C.M., and Swanson, R.A. 2000. Astrocyte glutamate transport: review of properties, regulation, and physiological functions. Glia 32:1-14.

8. Rose, C.R., Waxman, S.G., and Ransom, B.R. .1998. Effects of glucose deprivation, chemical hypoxia, and simulated ischemia on $\mathrm{Na}^{+}$homeostasis in rat spinal cord astrocytes. J. Neurosci. 18:3554-3562.

9. Jabaudon, D., Scanziani, M., Gähwiler, B.H. and Gerber, U. 2000. Acute decrease in net glutamate uptake during energy deprivation. Proc. Natl. Acad. Sci. USA 97:5610-5615.

10. Takahashi, S., Shibata, M., Gotoh, J. and Fukuuchi, Y. 2000. Astroglial cell death induced by excessive influx of sodium ions. Eur. J. Pharmacol. 408:127-135.

11. Kawahara, K., Hosoya, R., Sato, H., Tanaka, M., Nakajima, T. and Iwabuchi, S. 2002. Selective blockade of astrocytic glutamate transporter GLT-1 with dihydrokainate prevents neuronal death during ouabain treatment of astrocyte/neuron co-cultures. Glia 40:337-349.

12. Kawahara, K., Yanoma, J., Tanaka, M., Nakajima, T. and Kosugi, T. 2004. Nitric oxide produced during ischemia is toxic but crucial to preconditioning-induced ischemic tolerance of neurons in culture. Neurochem. Res. 29:797-804.

13. Kawahara, K., Kosugi, T., Tanaka, M., Nakajima, T. and Yamada, T. 2005. 
Reversed operation of glutamate transporter GLT-1 is crucial to the development of preconditioning-induced ischemic tolerance of neurons in neuron/astrocyte cocultures. Glia 49:349-359.

14. Kawahara, K., Abe, R., Yamauchi, Y. and Kohashi, M. 2002. Fluctuations of contraction rhythm during simulated ischemia/reperfusion in cultured cardiac myocytes from neonatal rats. Biol. Rhythm. Res. 33:339-350.

15. Maguire, G., Simko, H., Weinreb, R.N. and Ayoub, G. 1998. Transport-mediated release of endogenous glutamate in the vertebrate retina. Pflügers. Arch. 436:481484.

16. Innocenti, B., Parpura, V., and Haydon, P.G. 2000. Imaging extracellular waves of glutamate during calcium signaling in cultured astrocytes. J. Neurosci. 20:18001808.

17. Goldberg, M.P., and Choi, D.W. 1993. Combined oxygen and glucose deprivation in cortical cell culture: calcium-dependent and calcium-independent mechanisms of neuronal injury. J. Neurosci. 13:3510-3524.

18. Zelenaia, O., Schlag, B.D., Gochenauer, G.E., Ganel, R., Song, W., Beesley, J.S., Grinspan, J.B., Rothstein, J.D. and Robinson, M.B. 2000. Epidermal growth factor receptor agonists increase expression of glutamate transporter GLT-1 in astrocytes through pathways dependent on phosphatidylinositol 3-kinase and transcroption 
factor NF-кB. Mol. Pharmacol. 57:667-678.

19. Figiel, M., Maucher, T., Rozyczka, J., Bayatti, N. and Engele, J. 2003. Regulation of glial glutamate transporter expression by growth factors. Exp. Neurol. 183:124135.

20. Figiel, M., and Engele, J. 2000. Pituitary adenylate cyclase-activating polypeptide (PACAP), a neuron-derived peptide regulating glial glutamate transport and metabolism. J. Neurosci. 20:3596-3605.

21. Robinson, M.B. 1998. The family of sodium-dependent glutamate transporters: a focus on the GLT-1 / EAAT2 subtype. Neurochem. Int. 33:479-491.

22. Rao, V.L., Dogan, A., Bowen, K.K., Todd, K.G. and Dempsey, R.J. 2001. Antisense knockdown of the glial glutamate transporter GLT-1, but not the neuronal glutamate transporter EAAC1, exacerbates transient focal cerebral ischemia-induced neuronal damage in rat brain. J. Neurosci. 21:1876-1883.

23. Satoh, H., Ginsburg, K.S., Qing, K., Terada, H., Hayashi, H. and Bers, D.M. 2000. KB-R7943 block of $\mathrm{Ca}^{2+}$ influx via $\mathrm{Na}^{+} / \mathrm{Ca}^{2+}$ exchange does not alter twitches or glycoside inotropy but prevents $\mathrm{Ca}^{2+}$ overload in rat ventricular myocytes. Circulation 101:1441-1446.

24. Nedergaad, M. 1994. Direct signaling from astrocytes to neurons in cultures of 
mammalian brain cells. Science 263:1768-1771.

25. Florio, T., Grimaldi, M., Scorziello, A., Salmona, M., Bugiani, O., Tagliavini, F., Forloni, G. and Schettini, A. 1996. Intracellular calcium rise through L-type calcium channels, as molecular mechanism for prion protein fragment 106-126induced astroglial proliferation. Biochem. Biophys. Res. Com. 228:397-405.

26. Quandt, F.N., and Macvicar, B.A. 1986. Calcium activated potassium channels in cultured astrocytes. Neuroscience 19:29-41.

27. Parpura, V., Basarsky, T.A., Liu, F., Jeftinija, S. and Haydon, P.G. 1994. Glutamate-mediated astrocyte-neuron signalling. Nature 369:744-747.

28. Arakawa, N., Sakaue, M., Yokoyama, I., Hashimoto, H., Koyama, Y., Baba, A. and Matsuda, T. 2000. KB-R7943 inhibits store-operated $\mathrm{Ca}^{2+}$ entry in cultured neurons and astrocytes. Biochem. Biophys. Res. Com. 279:354-357.

29. Mitani, A., and Tanaka, K. 2003. Functional changes of glial glutamate transporter GLT-1 during ischemia: an in vivo study in the hippocampal CA1 of normal mice and mutant mice lacking GLT-1. J. Neurosci. 23:7176-7182.

30. Rothstein, J.D., Dykes-Hoberg, M., Pardo, C.A., Bristol, L.A., Jin, L., Kuncle, R.W., Kanai, Y., Hediger, M.A., Wang, Y., Schielke, J.P. and Welty, D.F. 1996. Knockout of glutamate transporters reveals a major role for astroglial transport in 
excitotoxicity and clearance of glutamate. Neuron 16:675-686.

31. Tanaka, K., Watase, K., Manabe, T., Yamada, K., Watanabe, M., Takahashi, K., Iwama, H., Nishikawa, T., Ichihara, N., Kikuchi, T., Okuyama, S., Kawashima, N., Hori, S., Takimoto, M. and Wada, K. 1997. Epilepsy and exacerbation of brain injury in mice lacking the glutamate transporter GLT-1. Science 276:1699-1702.

32. Sontheimer, H. and Ritchie, J.M. 1995. Voltage-gated Sodium and Calcium Channels., Kettenmann, H. and Ransom, B.R., Neuroglia. Oxford University Press, New York, 202-220.

33. Choi, D.W., and Rothman, S.M. 1990. The role of glutamate neurotoxicity in hypoxic-isocheims neuronal death. Annu. Rev. NeuroSci. 13:171-182. 
Figure legends

Fig. 1

Oxygen/glucose deprivation (OGD)-induced reverse mode operation of astrocytic glutamate transporter GLT-1 causes massive excitotoxic neuronal death in neuron/astrocyte mixed cultures. Figures $A 1, A 2$, and $A 3$ show the results of immunostaining of the mixed cultures using anti-MAP-2, anti-GFAP, and anti-GLT-1 antibodies, respectively. Photomicrographs B, C, D and E show the phase-contrast and nuclei staining images of sham-treated (B), OGD for 90 min (C), treatment with AP5 during OGD (D), and treatment with DHK during OGD $(E)$, respectively. Photomicrographs B1, C1, D1 and E1 show the cultures before exposure to OGD, whereas B2, C2, D2 and E2 illustrate their state $24 \mathrm{~h}$ after the exposure, respectively. Cell nuclei were stained with bisbenzimide (Hoechst 33342) and propidium iodide (PI) (B3-E3). Figure F shows a statistical comparison of the survival rate of neurons. The scale bar indicates $100 \mu \mathrm{m}$. Data are expressed as the mean+SD ( $n=4$ different cultures). ${ }^{*} p<0.01$. Abbreviations: Sham, sham-treatment with normal EBSS for 90 min; OGD, oxygen/glucose deprivation for 90 min; OGD+AP5, treatment with AP5 (100 $\mu \mathrm{M})$ during OGD; OGD+DHK, treatment with DHK $(200 \mu \mathrm{M})$ during OGD.

Fig. 2

Increased GLT-1 expression in PACAP-treated cultured astrocytes and oxygen/glucose deprivation (OGD)-induced reverse mode operation of GLT-1. 
Figures $A 1, A 2, A 3$, and $A 4$ show the results of immunostaining of the astrocyte-enriched cultures using anti-GFAP (A1), anti-MAP-2 (A2), anti-GLT-1 in sham-treated astrocytes (A3), and anti-GLT-1 antibodies in PACAP-treated astrocytes (A4), respectively. Figures B-D show the changes in the extracellular concentration of glutamate during OGD in cultured astrocytes. Photomicrographs B1, C1, and D1 show the phase-contrast images of the astrocyte-enriched cultures. Figures B2-B4, C2-C4, and D2-D4 illustrate the pseudo-color representation of $\mathrm{NADH}$ fluorescence reflecting the extracellular concentration of glutamate when the sham-treated astrocyte cultures (B2-B4), the astrocyte cultures treated with PACAP for $24 \mathrm{~h}(\mathrm{C} 2-\mathrm{C} 4)$, and the PACAPtreated cultures with DHK $(400 \mu \mathrm{M})$ treatment (D2-D4), were exposed to OGD, respectively. Figures $\mathrm{B} 2, \mathrm{C} 2$, and D2 represent the fluorescence at 5 min after the onset of OGD. Figures B3, C3, D3 and B4, C4, D4 represent the fluorescence at 2 and $4 \mathrm{~h}$ after the onset of OGD, respectively. Western blot analysis of GLT-1 (E1) and actin (E2) in the sham-treated and PACAP-treated (24 h after PACAP treatment) astrocyte-enriched cultures. Figure F shows the quantification of GLT-1 (F1), and actin (F2). The expression levels are normalized with the mean expression level in the sham-treated cultures. Data are expressed as the mean $+S D(n=4$ different cultures). * $p<0.01$. Figure $G$ shows a time course of the change in the NADH fluorescence ratio during OGD in the sham-treated cultures (blue), in the PACAP-treated cultures (pink), and in the PACAP-treated cultures with DHK treatment (red), respectively. The OGD begins at 0 min. Vertical bars indicate + or -SD. Figures $\mathrm{H} 1, \mathrm{H} 2$, and $\mathrm{H} 3$ show a statistical comparison of the relative fluorescence intensity at 2, 3, and $4 \mathrm{~h}$ 
after the onset of OGD, respectively. The scale bar indicates $100 \mu \mathrm{m}$. Data are expressed as the mean+SD ( $n=4$ different cultures). * $p<0.01$. Abbreviations: PACAP-treated, astrocytes treated with PACAP $(1 \mu \mathrm{M})$ for $24 \mathrm{~h}$; PACAP-treated + DHK, PACAP-treated astrocytes with treatment with DHK $(400 \mu \mathrm{M})$ during OGD; F0, fluorescence intensity just before the onset of OGD; F, fluorescence intensity during OGD.

Fig. 3

Oxygen/glucose deprivation (OGD)-induced reverse mode operation of astrocytic glutamate transporter GLT-1 contributes to the survival of astrocytes against ischemic insult. Data are expressed as the mean+SD ( $n=4$ different cultures). * $p<0.01$. Abbreviations: sham-treated (OGD), sham-treated astrocytes exposed to $4 \mathrm{~h}$ OGD; PACAP-treated (OGD), PACAP-treated astrocytes exposed to 4 h OGD; PACAP-treated + DHK (OGD), PACAP-treated astrocytes with treatment with DHK $(400 \mu \mathrm{M})$ during 4 h OGD; sham-treated + DHK (OGD), sham-treated astrocytes with treatment with DHK (400 $\mu \mathrm{M})$ during 4 h OGD; sham-treated + DHK, sham-treated astrocytes with treatment with DHK $(400 \mu \mathrm{M})$ for $4 \mathrm{~h}$ without OGD.

Fig. 4 
Changes in the concentration of intracellular $\mathrm{Na}^{+}$and $\mathrm{Ca}^{2+}$ in cultured astrocytes during oxygen/glucose deprivation (OGD). Photomicrographs A1, B1, C1, and D1 show phase-contrast images of the astrocyte-enriched cultures. Figures A2-D2 and A4-D4 show the SBFI and Fluo-4 fluorescence 5 min after the onset of OGD, respectively. Figures A3-D3 and A5-D5 show the SBFI and Fluo-4 fluorescence $4 \mathrm{~h}$ after the onset of OGD, respectively. Figures $\mathrm{A}, \mathrm{B}, \mathrm{C}$, and D indicate sham-treated, treated with PACAP for $24 \mathrm{~h}$, PACAP-treated with DHK $(400 \mu \mathrm{M})$, and sham-treated with KB-R7943 $(5 \mu \mathrm{M})$, respectively. It should be noted that the OGD-induced rise in both intracellular $\mathrm{Na}^{+}$and $\mathrm{Ca}^{2+}$ was markedly suppressed in the PACAP-treated cultured astrocytes (B3 and B5). Treatment with KB-R7943, a blocker of the reversed $\mathrm{Na}^{+}-\mathrm{Ca}^{2+}$ exchanger (NCX), markedly reduced the OGD-induced rise in $\mathrm{Ca}^{2+}(\mathrm{D} 5)$, but did not reduce the rise in $\mathrm{Na}^{+}(\mathrm{D} 3)$. The scale bar indicates $100 \mu \mathrm{m}$.

Fig. 5

Time courses of the changes in intracellular $\mathrm{Na}^{+}$and $\mathrm{Ca}^{2+}$ in cultured astrocytes during oxygen/glucose deprivation (OGD). Figure A1 shows the changes in the ratio of SBFI fluorescence $\left(\mathrm{F}_{340} / \mathrm{F}_{380}\right)$. Figure $\mathrm{B} 1$ shows the changes in the ratio of Fluo-4 fluorescence (F/Fo). Both in Figures A1 and B1, all values are the mean of the fluorescence at each time point from 6 cells with + or $-\mathrm{SD}$. Figures $\mathrm{A} 2$ and $\mathrm{B} 2$ indicate the statistical comparisons of the SBFI and Fluo-4 fluorescence intensities $4 \mathrm{~h}$ after the onset of OGD, respectively. Data are expressed as the mean+SD ( $n=12$ cells from 2 different cultures). * 
$\mathrm{p}<0.01$. Figure $\mathrm{C}$ indicates astrocytic damage at $20 \mathrm{~h}$ after the end of $4 \mathrm{~h}$ OGD with treatment with KB-R7943. Abbreviations: sham-treated (OGD), shamtreated astrocytes exposed to $4 \mathrm{~h}$ OGD; sham-treated (OGD, no indicator), sham-treated astrocytes exposed to $4 \mathrm{~h}$ OGD without loading of cells with fluorescence indicators; PACAP-treated (OGD), PACAP-treated astrocytes exposed to $4 \mathrm{~h}$ OGD; PACAP-treated (OGD, no indicator), PACAP-treated astrocytes exposed to $4 \mathrm{~h}$ OGD without loading of cells with fluorescence indicators; PACAP-treated + DHK (OGD), PACAP-treated astrocytes with treatment with DHK $(400 \mu \mathrm{M})$ during $4 \mathrm{~h}$ OGD; sham-treated + KBR (OGD), sham-treated astrocytes with treatment with KB-R7943 (5 $\mu \mathrm{M})$ during OGD. 
Fig. 1
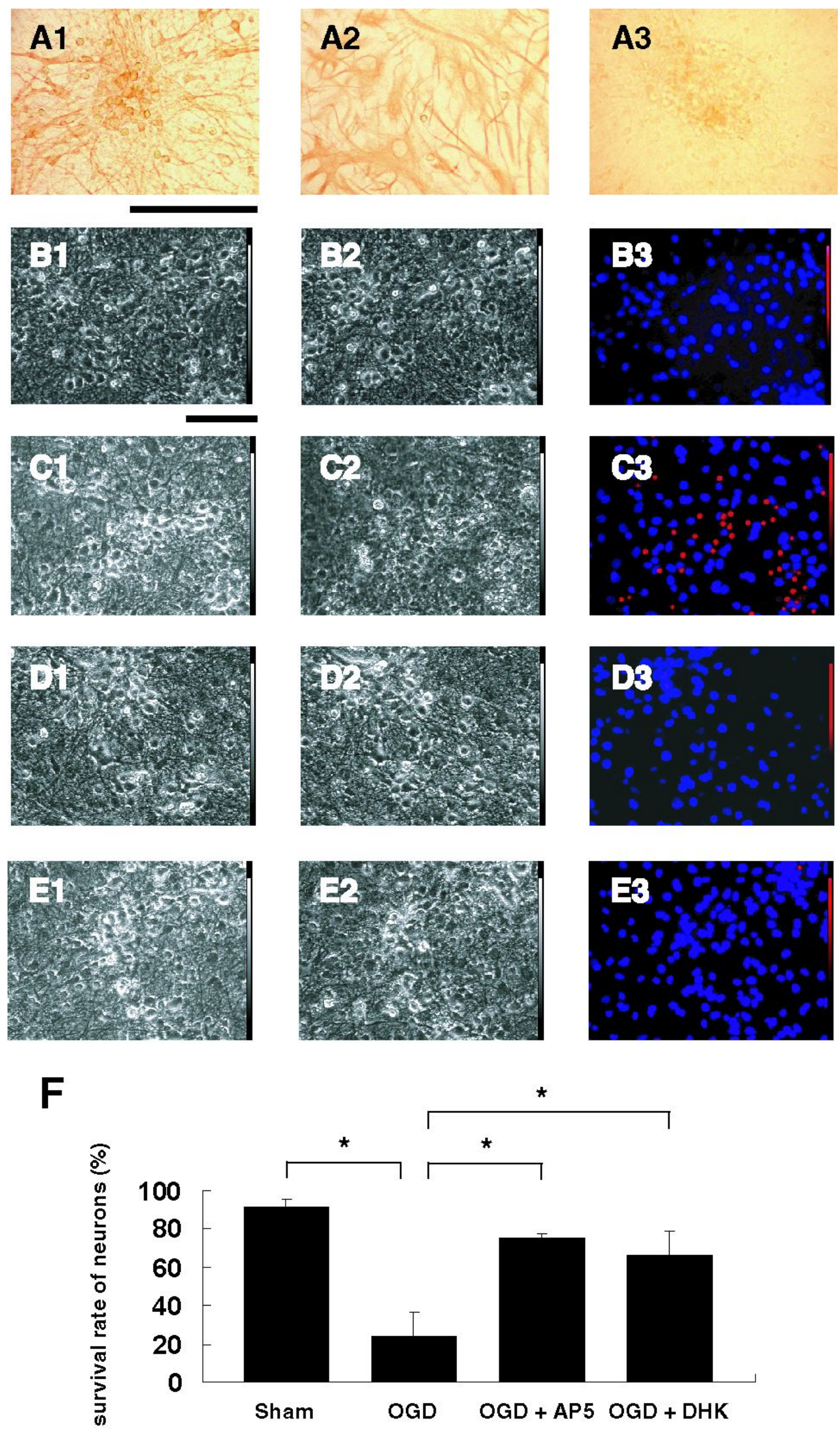
Fig. 2

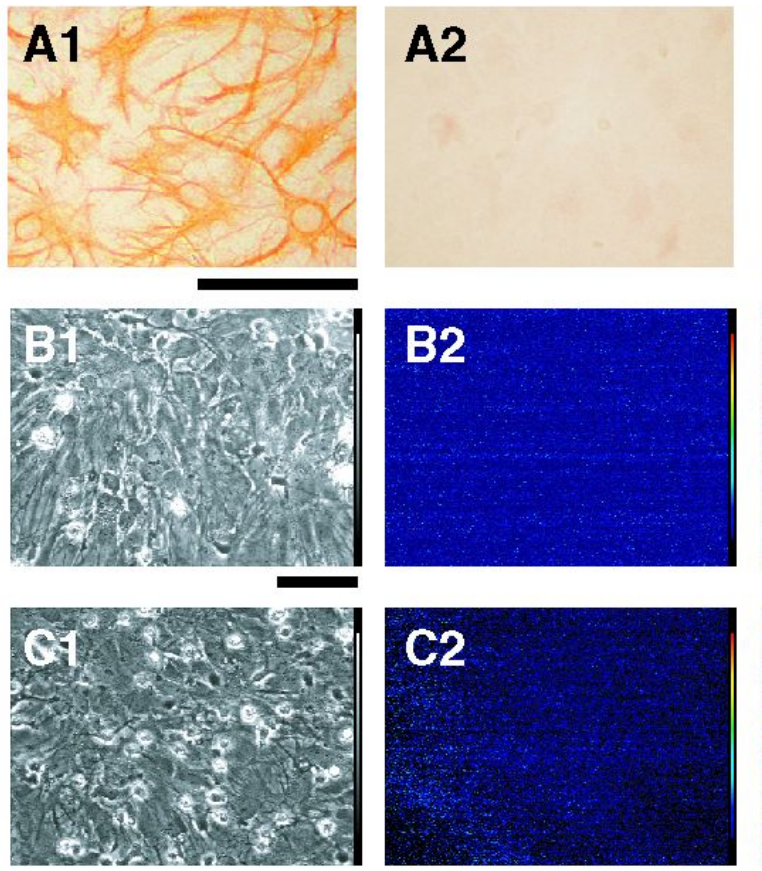

A3
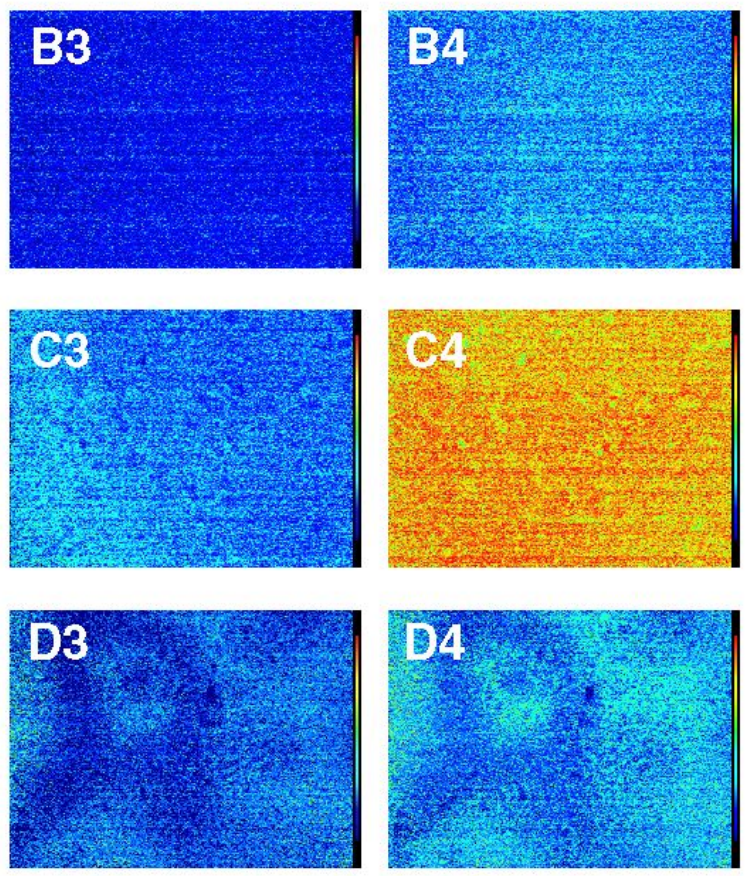

A4
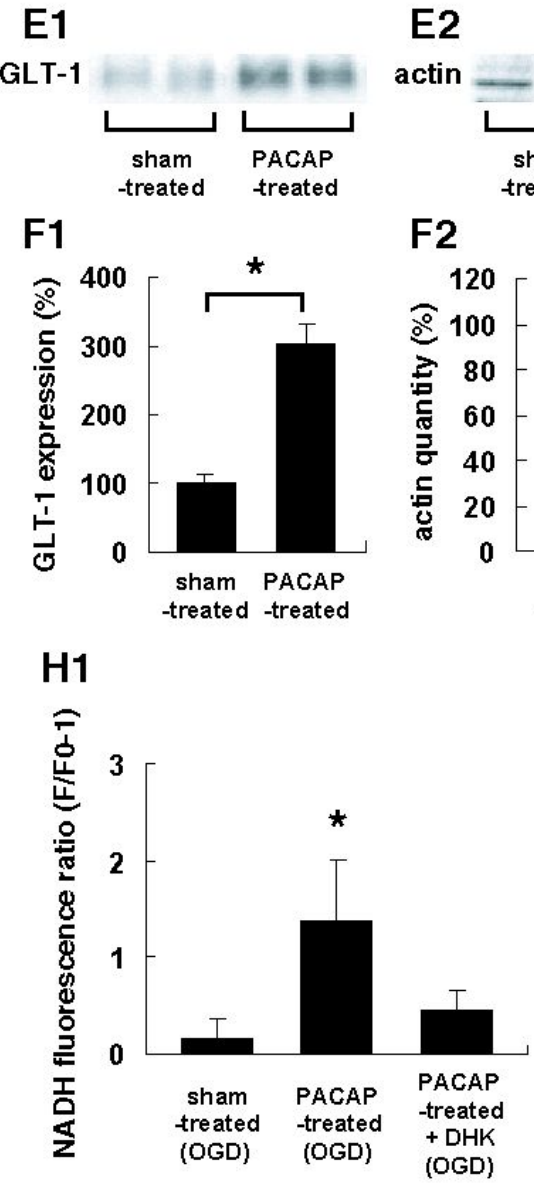

\section{E2}

F2
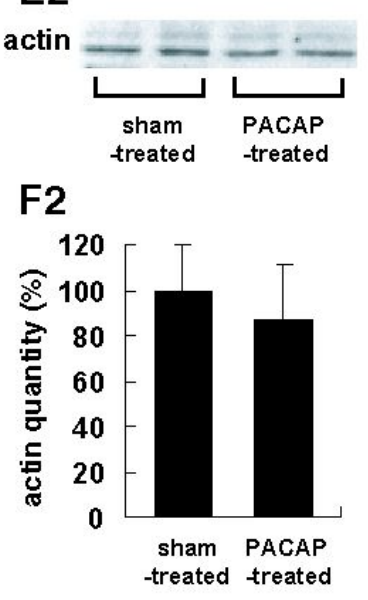

$\mathrm{H} 2$

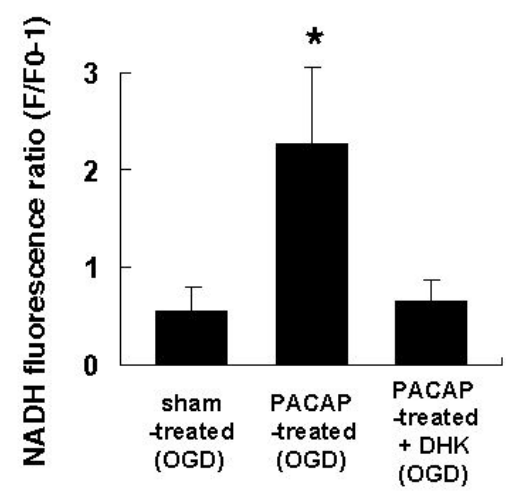

G

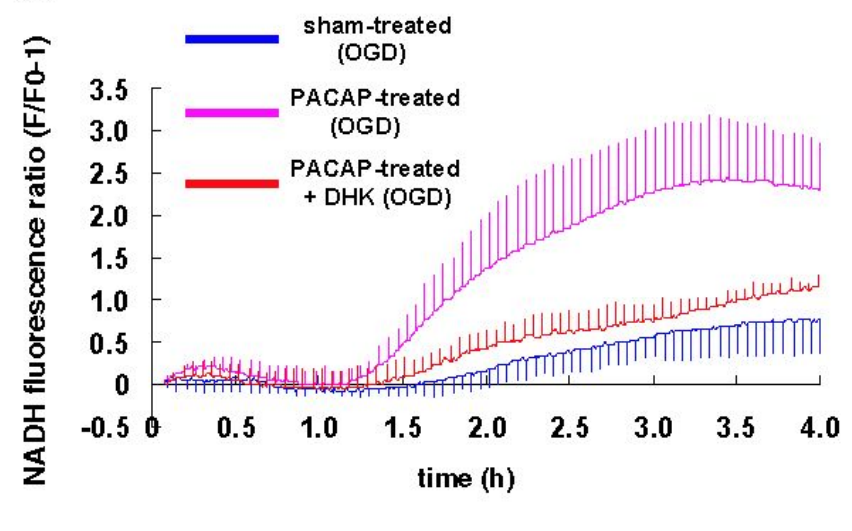

\section{H3}

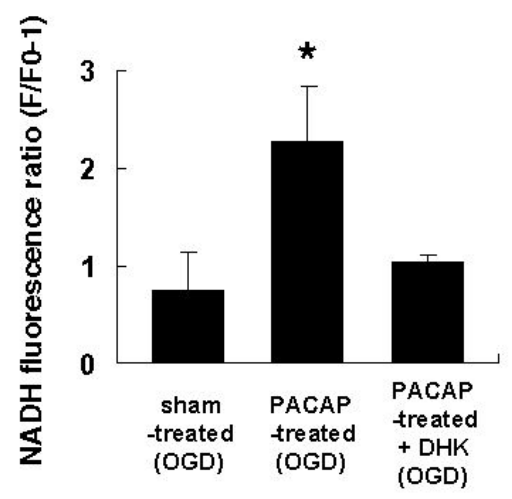


Fig. 3

A

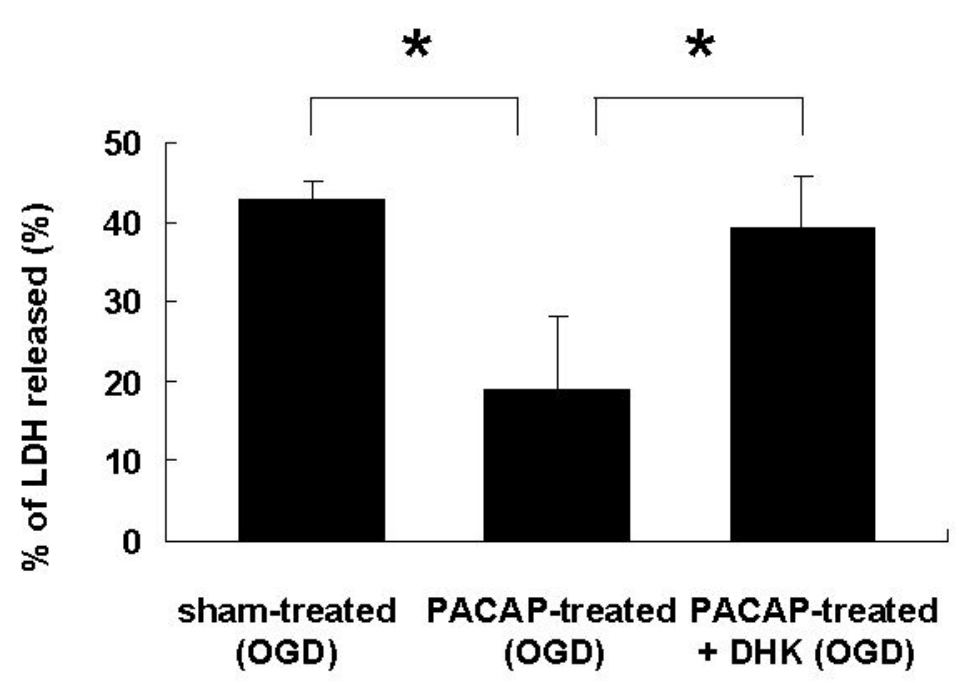

B

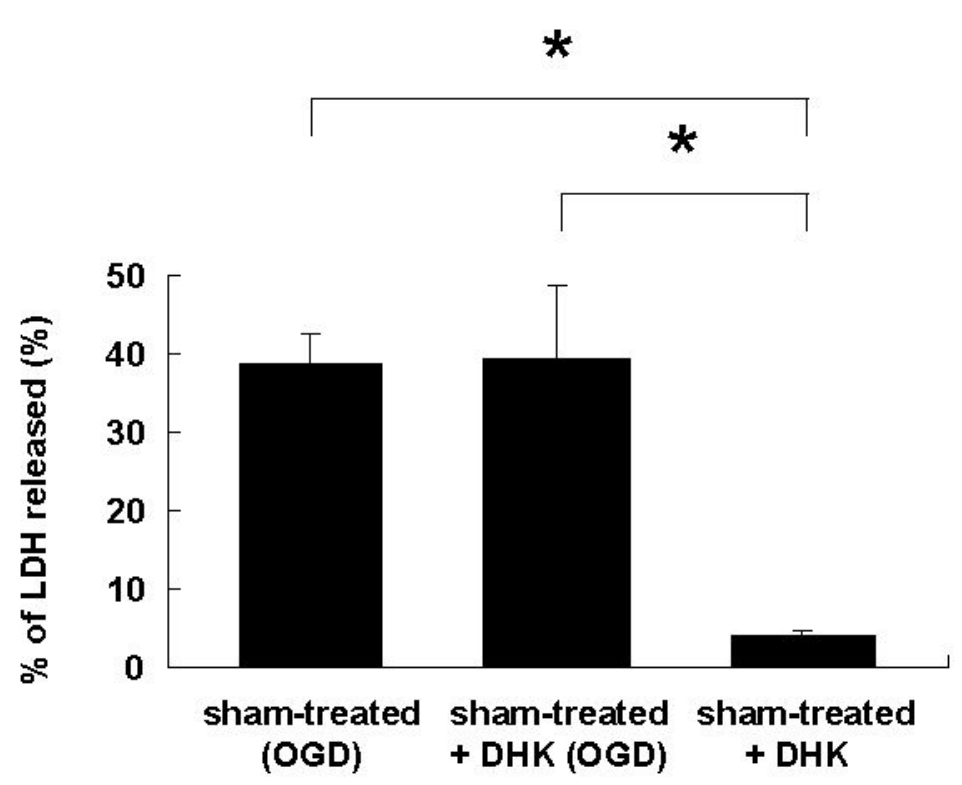


Fig. 4
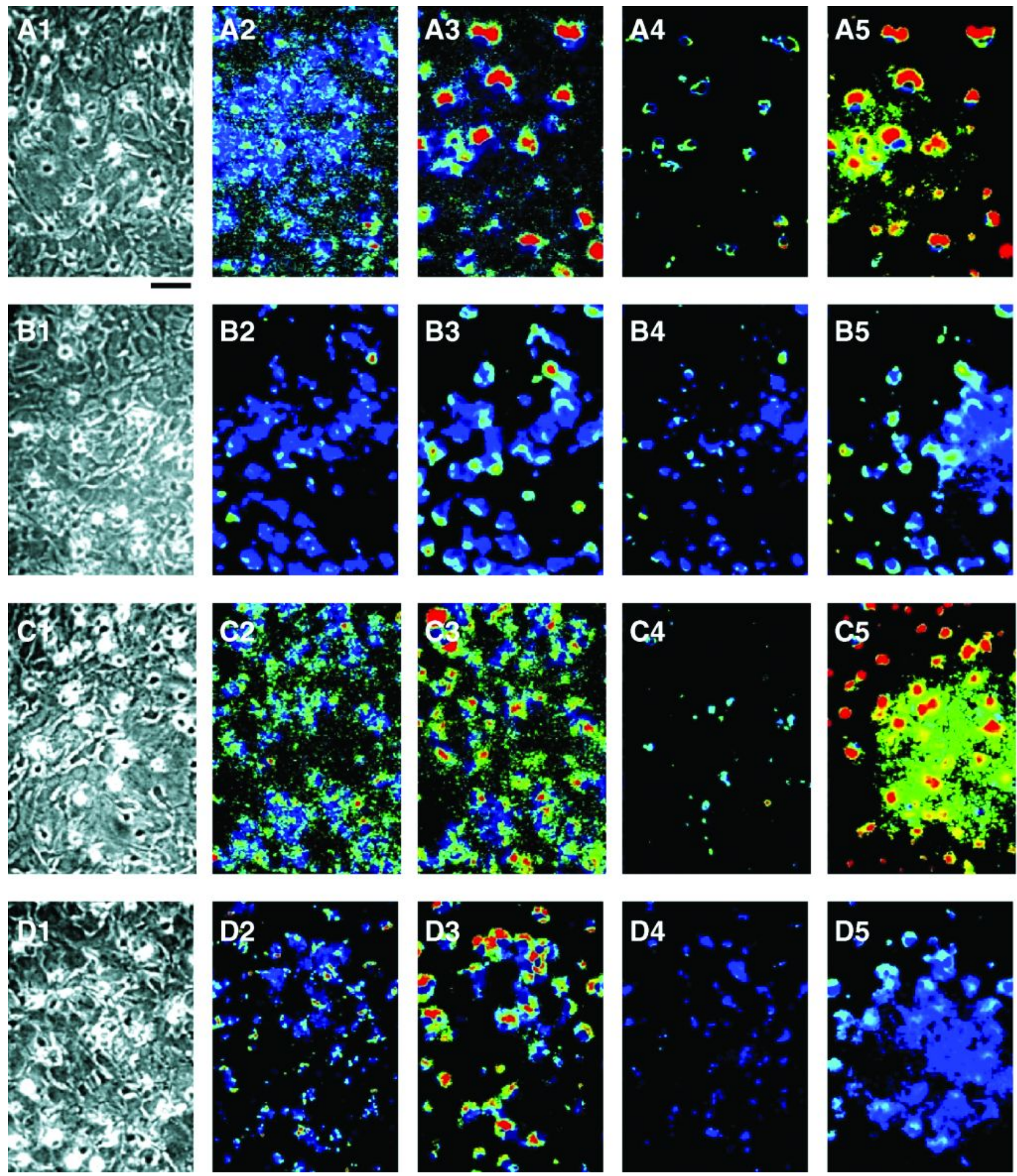
Fig. 5

A1

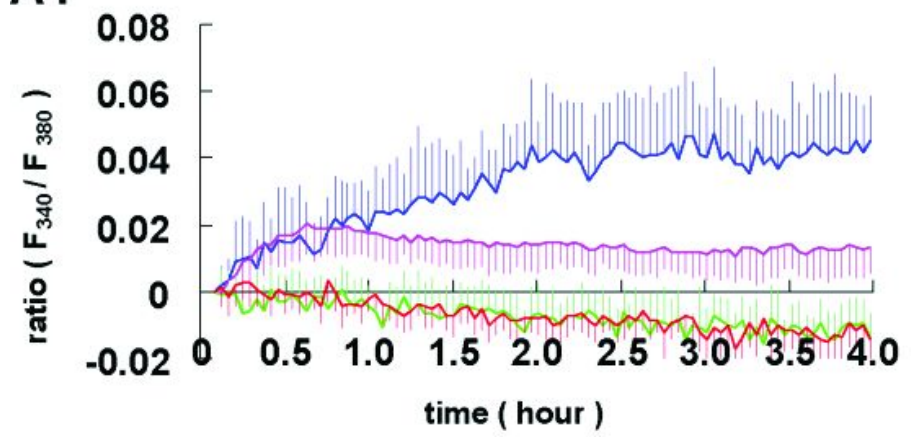

sham-treated (OGD)

sham-treated (OGD, no indicator)
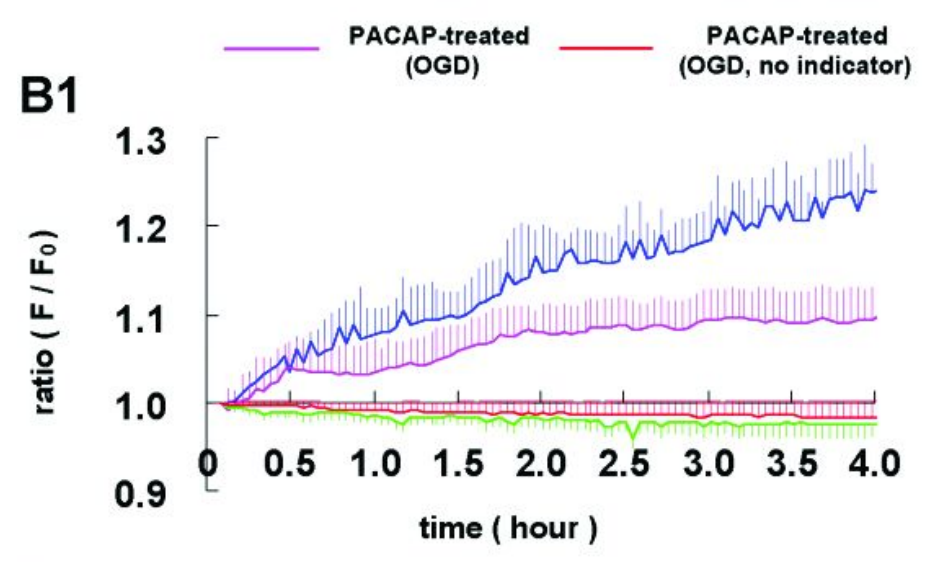

C

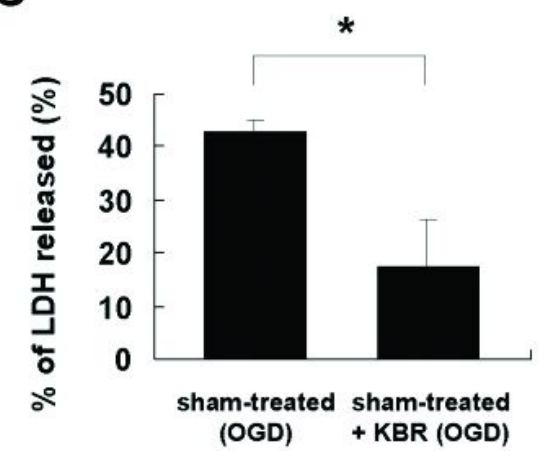

A2

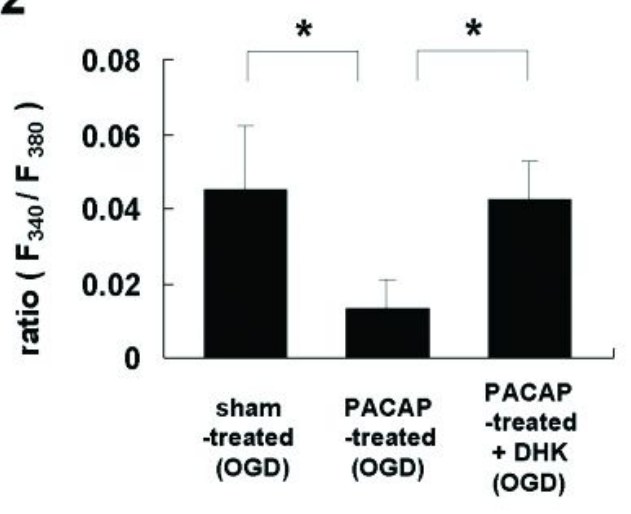

B2

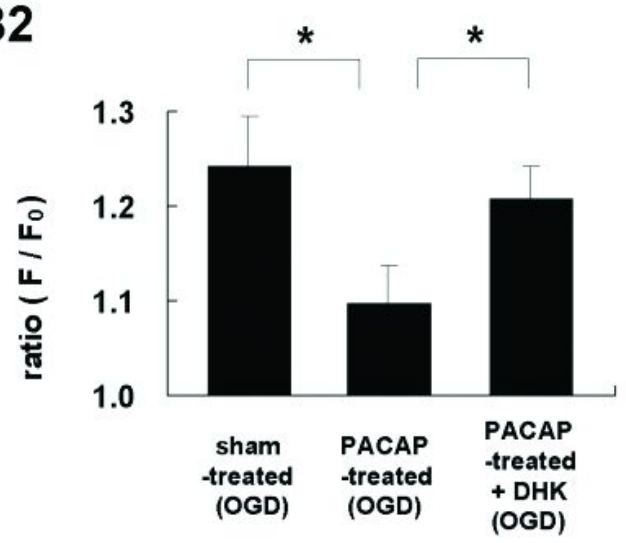

